\title{
Optimal Proportional-Integral Guidance with Reduced Sensitivity to Target Maneuvers
}

\author{
Shaoming He and Chang-Hun Lee*
}

\begin{abstract}
This paper proposes a new optimal guidance law based on proportional-integral (PI) concept to reduce the sensitivity to unknown target maneuvers. Compared to existing PI guidance laws, the proposed guidance command is derived in the optimal control framework while guaranteeing finite time convergence. The kinematics equation with respect to the zero-effort-miss (ZEM) is utilized and the integral ZEM is augmented as a new system state. The proposed guidance law is derived through the Schwarz's inequality method. The closed-form solution of proposed guidance law is presented to provide better insight of its properties. Additionally, the working principle of the integral command is investigated to show why the proposed guidance law is robust against unknown target accelerations. The analytical results reveal that the proposed optimal guidance law is exactly the same as an instantaneous direct model reference adaptive guidance law with a pre-specified reference model. The potential significance of the obtained results is that it can provide a point of connection between PI guidance laws and adaptive guidance laws. Therefore, it allows us to have better understanding of the physical meaning of both guidance laws and provides the possibility in designing a new guidance law that takes advantages of both approaches. Finally, the performance of the guidance law developed is demonstrated by nonlinear numerical simulations with extensive comparisons.
\end{abstract}

\section{Index Terms}

Optimal guidance law, PI control, Target maneuver, Schwarz's inequality

\section{INTRODUCTION}

The conventional proportional navigation guidance (PNG) law [1], [2] has been extensively applied to various missile systems as the terminal homing guidance law over the past several decades. The key features driving the success of PNG are its simplicity and efficiency of implementation. The basic idea of PNG is that it generates a guidance command to nullify the line-of-sight (LOS) rate, forcing the missile to follow the collision course. Moreover, the PNG with a specific navigation constant, $N=3$, is known as an energy optimal homing guidance law. However, it is well-known that the performance of conventional PNG degrades drastically with the increase of target maneuverability. Also, as shown in [3]-[8], the performance of conventional PNG, when against a maneuvering target, is far away from the optimal one. As a remedy, the augmented PNG (APNG) law was proposed by

Shaoming He and Chang-Hun Lee are with the School of Aerospace, Transport and Manufacturing, Cranfield University, Cranfield MK43 OAL, UK.

*Corresponding Author. Email: lckdgns@gmail.com or to reuse any copyrighted component of this work in other works must be obtained from the IEEE. 
introducing a target acceleration rejection term in the PNG guidance command [1]. Obviously, the information on target acceleration is required for the implementation of APNG [1], [9]. In practice, unfortunately, it is difficult to obtain the information on target acceleration since it is uncertain in nature. A filtering algorithm can be utilized to estimate the unknown target acceleration for the implementation of APNG. However, APNG with estimated target acceleration cannot provide good performance in some engagement scenarios as shown in [10].

In order to tackle the issue of maneuvering target interception, adaptive control technique is found to be widelyaccepted in robust guidance law design. The basic idea of adaptive guidance is that it leverages a direct or indirect adaptive law to estimate the unknown target maneuver and feedback this information to the guidance command to partially reject the effect of target maneuvers. In [11], the authors proposed a nonlinear adaptive guidance law by considering the autopilot dynamics to compensate for the unknown target maneuver. The numerical results revealed that the presented guidance law was better than PNG and sliding mode guidance law. Further extension of [11] was made in [12] through an observer design to estimate the higher-order LOS rate derivatives for practical applications. A systematic backstepping in conjunction with adaptive control was suggested in [13] to design robust guidance law with input saturation constraint. A new fast adaptive guidance law based on the optimal modification was proposed in [14] to intercept highly maneuverable targets. A novel integrated guidance and control was developed for maneuvering target interception using adaptive control technique in [15], where a nonlinear observer was designed to estimate both uncertainties and unknown system states. Recently, adaptive control technique was also leveraged for target maneuver estimation in guidance law design to cater for impact angle constraint [16], [17].

In the field of control technology, it is well-understood that the concept of PI control, apart from the adaptive control technique, is a simple and an effective way to attenuate the effect of external disturbances. Based on this aspect, guidance laws based on PI-control were proposed recently in [18], [19]. The rationale behind this approach is that if the unknown target acceleration is considered as an external disturbance of the guidance system from the control perspective, then the integral term in the guidance command can help reduce the sensitivity to the unknown target acceleration. Recent works regarding PI guidance in [18], [19] revealed that it can significantly improve the guidance performance for a maneuvering target in terms of miss distance compared to the conventional PNG. However, the guidance law, proposed in [18], disregards what is the optimal PI guidance law in terms of a meaningful performance index. Although the authors in [19] have attempted to find out the optimal PI guidance command, the solution obtained was incomplete: the guidance gains associated with PI terms were not given by explicit form and should be tuned at each time step. In both PI guidance laws [18], [19], the finite time convergence was not strictly guaranteed. Moreover, there is no rigorous explanation regarding the working principle of PI guidance laws. Namely, in these approaches, there still remain some concerns that how the PI guidance laws can reduce the sensitivity to the unknown target acceleration and what is the physical meaning of the integral command part. In practice, such information is important in ensuring confidence in the performance and reliability of the guidance law when implementing it in a real system.

Motivated by the above observations, this paper aims to propose a new PI guidance law based on optimal control framework while guaranteeing finite time convergence. Additionally, this paper also aims to investigate the physical meaning of guidance laws based on the PI concept, especially for the interpretation of the integral term. To this end, 
unlike previous approaches [18], [19], which were based on the LOS rate format, the ZEM is selected as the state variable to be controlled. This selection allows us to formulate the guidance problem simply. In order to exploit the benefit of PI control, the integral of ZEM is introduced as an augmented state variable in the kinematic equation. The proposed optimal guidance law is derived by solving a predetermined linear quadratic (LQ) optimal guidance problem through the Schwarz's inequality approach [1], [20]. The obtained guidance command is given by a timevarying PI controller with respect to the ZEM. By introducing the relationship between the ZEM and the LOS rate, the proposed guidance command can be realized as the conventional PNG with an additional integral term of PNG command weighted by the time-to-go. The closed-form solution of the proposed guidance law is also derived to provide better insight of its properties. By using an inverse approach (i.e. directly differentiating the integral term of the proposed PI guidance law) and utilizing the instantaneous linear time-invariant system concept, it is shown that the proposed optimal PI guidance law is exactly the same as an instantaneous direct model reference adaptive guidance law with a specified reference model. In this analysis, the physical meaning of the proposed guidance law is clearly shown. Also, in a similar way, other PI guidance laws can be analyzed.

The main contributions of this paper are as twofolds. On one hand, in order to reduce the sensitivity against unknown target maneuver, we propose a new optimal PI guidance law that guarantees finite time convergence. On the other hand, the physical meaning of the PI guidance laws is analyzed by comparing it with adaptive guidance laws. This result provides us better understanding of the PI guidance and establishes a theoretical link between PI guidance and adaptive guidance. Accordingly, it suggests the possibility in designing a new guidance law that exploits the benefits of both approaches.

The rest of the paper is organized as follows. Sec. II presents some preliminaries and backgrounds of this paper. Sec. III derives the proposed optimal PI guidance law, followed by the theoretical analysis shown in Sec. IV. Finally, some numerical simulations and conclusions are offered.

\section{PROBLEM Formulation}

This section states the problem formulation of this paper. Before introducing the system kinematics, we make three basic assumptions as follows:

Assumption 1. Both thel missile and the target are assumed as ideal point-mass models.

Assumption 2. The engagement occurs in a 2-D plane.

Assumption 3. Both the missile and the target are flying with constant velocity.

Note that these assumptions are widely accepted in guidance law design for tactical missiles: (Assumption 1) Typical philosophy treats the guidance and control loops separately by placing the guidance kinematics in an outer-loop, generating guidance commands tracked by an inner dynamic control loop, also known as autopilot. (Assumption 2) It is assumed that the missile employs an ideal attitude control system that provides roll stabilization such that the guidance problem can be treated in two separate channels. Accordingly, homing engagement can be 


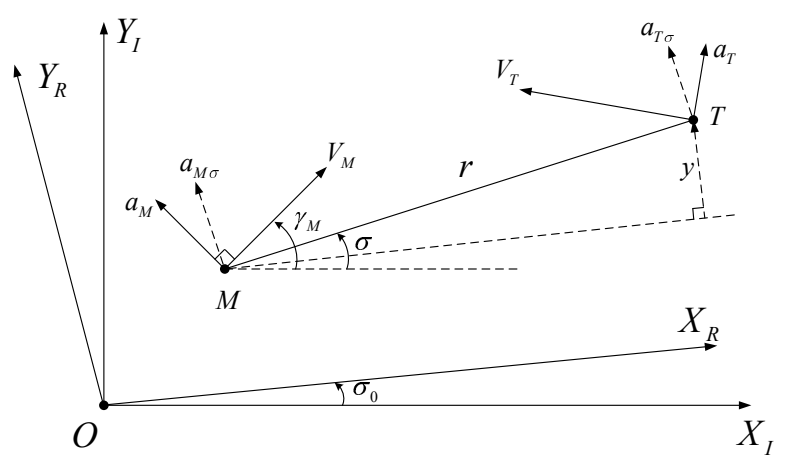

Fig. 1: The homing engagement geometry and parameter definitions.

treated as a 2-D problem. (Assumption 3) The vehicle's velocity is generally slowly varying and hence can be assumed as piece-wise constant.

\section{A. Missile-Target Relative Kinematics}

Figure 1 shows the planar homing engagement geometry in this study, where $M$ and $T$ denote the missile and target, respectively. The notation of $\left(X_{I}, Y_{I}\right)$ represents the inertial frame. The reference frame $\left(X_{R}, Y_{R}\right)$ is also introduced with the purpose of deriving the linearized engagement kinematics. The reference frame is rotated from the inertial frame by the initial LOS angle $\sigma_{0}$, which is the reference angle. The variables $\sigma$ and $\gamma$ stand for the LOS angle and flight path angle, respectively. The variable $r$ represents the relative distance between the missile and the target. The variable $y$ denote the relative lateral position between the missile and the target perpendicular to the $X_{R}$-direction. The variables $a_{M}$ and $a_{T}$ denote the missile and target accelerations normal to their velocity vectors, respectively. Dually, the variables $a_{M_{\sigma}}$ and $a_{T_{\sigma}}$ represent the missile and target accelerations normal to the LOS vector, respectively. From Fig. 1, the engagement kinematics equation with respect to the reference frame can be obtained as

$$
\begin{aligned}
& \dot{y}=v \\
& \dot{v}=a_{T_{\sigma}} \cos \left(\sigma-\sigma_{0}\right)-a_{M_{\sigma}} \cos \left(\sigma-\sigma_{0}\right)
\end{aligned}
$$

where $v$ represents the relative lateral velocity between the missile and the target perpendicular to $X_{R}$-direction.

During the terminal homing phase, the LOS angle does not deviate too much from the initial LOS in general. Therefore, the LOS angle with respect to the initial LOS (i.e., $\sigma-\sigma_{0}$ ) is small enough to linearize system Eq. (1) as

$$
\begin{aligned}
& \dot{y}=v \\
& \dot{v}=a_{T_{\sigma}}-a_{M_{\sigma}}
\end{aligned}
$$

The complementary equation that describes the relationship between $a_{M_{\sigma}}$ and $a_{M}$ is given by

$$
a_{M_{\sigma}}=a_{M} \cos \left(\gamma_{M}-\sigma\right)
$$

From Fig. 1, the LOS angle in the linearized engagement kinematics can be approximated as

$$
\sigma=\frac{y}{r}+\sigma_{0}
$$


Taking the time-derivative of Eq. (4) provides the LOS rate in the linearized engagement kinematics as

$$
\dot{\sigma}=\frac{y+v t_{g o}}{V_{c} t_{g o}^{2}}
$$

where $V_{c}$ denotes the closing velocity, and $t_{g o}$ the remaining flight time or the so-called time-to-go.

\section{B. Problem Definition}

Note that the engagement kinematics shown in Eq. (2) contains the unknown target acceleration. In order to provide a satisfactory guidance performance for intercepting maneuvering targets, a guidance law should be less sensitive to the unknown target accelerations. Accordingly, as mentioned in the introduction, the main purpose of this paper is to devise a new guidance law to reduce the sensitivity against the unknown target maneuver using PI-control concept. Different from existing PI guidance laws [18], [19], we formulate the aforementioned guidance problem based on the rigorous optimal control framework in order to consider optimality and finite time convergence simultaneously.

In guidance law design, it is well-known that the ZEM or the LOS rate should converge to zero in a finite time in order to guarantee a perfect interception. To this end, we consider the system equation in terms of ZEM because it makes the derivation of the proposed guidance law simple. In this study, let $z$ be the ZEM and the most widely used definition of ZEM [1] is adopted. Additionally, in order to avoid dependency of target acceleration information in a resultant guidance law, the ZEM definition in the absence of target acceleration is used. Accordingly, in the linearized engagement kinematics, the considered ZEM is given as

$$
z=y+v t_{g o}
$$

The ZEM, defined in Eq. (6), is then leveraged in guidance law design and the unknown target acceleration is considered as a disturbance for the guidance system to be rejected. From Eqs. (3) and (6), the time derivative of $z$ is given by

$$
\dot{z}=-t_{g o} a_{M \sigma}+t_{g o} a_{T \sigma}
$$

As shown in Eq. (7), the unknown target acceleration $a_{T \sigma}$ appears in the ZEM dynamics. Since $a_{T \sigma}$ is unknown, we neglect this disturbance term as

$$
\dot{z}=-t_{g o} a_{M \sigma}
$$

Instead, in order to reduce the sensitivity against the neglected term, we exploit the PI control concept as discussed before: we introduce the integration of ZEM, i.e., $\int z d t$, as an augmented state variable. For notation convenience, we define new variables $x_{1}$ and $x_{2}$ as

$$
x_{1}=\int z d t, \quad x_{2}=z
$$

By using these two variables, the system equations shown in Eqs. (6) and (8) can then be rewritten in a compact matrix form as

$$
\left[\begin{array}{c}
\dot{x}_{1} \\
\dot{x}_{2}
\end{array}\right]=\left[\begin{array}{ll}
0 & 1 \\
0 & 0
\end{array}\right]\left[\begin{array}{l}
x_{1} \\
x_{2}
\end{array}\right]+\left[\begin{array}{c}
0 \\
-t_{g o}
\end{array}\right] a_{M_{\sigma}}
$$


where the $a_{M_{\sigma}}$ is the guidance command to be designed.

Based on the system equation shown in Eq. (10), the proposed guidance law aims to minimize

$$
J=\frac{1}{2} \int_{t}^{t_{f}} R(\tau) a_{M_{\sigma}}^{2}(\tau) d \tau
$$

subject to the terminal constraints

$$
x_{1}\left(t_{f}\right)=0, \quad x_{2}\left(t_{f}\right)=0
$$

where $t_{f}$ represents the time of impact, $R(t)>0$ the arbitrary weighting function.

The constraint $x_{2}\left(t_{f}\right)=0$ is the zero terminal ZEM, ensuring the interception of the target, and the constraint $x_{1}\left(t_{f}\right)=0$ is leveraged to minimize the cumulative ZEM so as to reduce the sensitivity to external disturbances. Note that if we only enforce the constraint $x_{2}\left(t_{f}\right)=0$ in guidance law design, the resulting formulation is the well-justified PNG law. In the next section, the proposed guidance law will be determined by solving the optimal guidance problem, described by Eqs. (11) and (12).

\section{Derivation of the Proposed Optimal Guidance LaW}

In this section, we first derive the proposed guidance law using Schwarz's inequality approach and then discuss the implementation issue of the proposed guidance law.

\section{A. Derivation of the Proposed Optimal Guidance}

As shown in Eq. (10), the system equation contains the time-varying nonlinear term due to time-to-go (i.e., $\left.t_{g o}\right)$. Accordingly, because of this nonlinearity, it is relatively difficult to apply the standard optimal control approach to find the analytical guidance command. We thereby seek to solve the preceding optimal control problem through Schwarz's inequality approach [1]. According to the linear control theory, the general solution of Eq. (10) can be expressed as

$$
\begin{aligned}
& x_{1}\left(t_{f}\right)=f_{1}-\int_{t}^{t_{f}} h_{1}(\tau) a_{M_{\sigma}}(\tau) d \tau \\
& x_{2}\left(t_{f}\right)=f_{2}-\int_{t}^{t_{f}} h_{2}(\tau) a_{M_{\sigma}}(\tau) d \tau
\end{aligned}
$$

where

$$
\begin{array}{ll}
f_{1} \triangleq x_{1}(t)+\left(t_{f}-t\right) x_{2}(t), & h_{1} \triangleq\left(t_{f}-\tau\right)^{2} \\
f_{2} \triangleq x_{2}(t), & h_{2} \triangleq t_{f}-\tau
\end{array}
$$

Imposing the terminal constraints Eq. (12) on Eq. (13) gives

$$
\begin{aligned}
& f_{1}=\int_{t}^{t_{f}} h_{1}(\tau) a_{M_{\sigma}}(\tau) d \tau \\
& f_{2}=\int_{t}^{t_{f}} h_{2}(\tau) a_{M_{\sigma}}(\tau) d \tau
\end{aligned}
$$

Obviously, the considered problem is an under-actuation problem, in which two constraints are required to be satisfied with only one control input. To this end, a Lagrange multiplier $\lambda$ is introduced to merge these two constraints as

$$
f_{1}-\lambda f_{2}=\int_{t}^{t_{f}}\left[h_{1}(\tau)-\lambda h_{2}(\tau)\right] a_{M_{\sigma}}(\tau) d \tau
$$


Introducing a slack variable $R(t)$ renders Eq. (16) to

$$
f_{1}-\lambda f_{2}=\int_{t}^{t_{f}}\left[h_{1}(\tau)-\lambda h_{2}(\tau)\right] R^{-1 / 2}(\tau) R^{-1 / 2}(\tau) a_{M_{\sigma}}(\tau) d \tau
$$

Applying the Schwarz's inequality to the preceding equation yields

$$
\frac{\left(f_{1}-\lambda f_{2}\right)^{2}}{2 \int_{t}^{t_{f}}\left[h_{1}(\tau)-\lambda h_{2}(\tau)\right] R^{-1}(\tau) d \tau} \leq \frac{1}{2} \int_{t}^{t_{f}} R^{-1}(\tau) a_{M_{\sigma}}^{2}(\tau) d \tau
$$

which gives a lower bound of the performance index. According to the Schwarz's inequality, the equality of Eq. (18) holds if and only if there exists a constant $C$ such that

$$
a_{M_{\sigma}}(\tau)=C\left[h_{1}(\tau)-\lambda h_{2}(\tau)\right] R^{-1}(\tau)
$$

Substitution of Eq. (19) into Eq. (15) results in

$$
f_{1}=C \int_{t}^{t_{f}} h_{1}(\tau)\left[h_{1}(\tau)-\lambda h_{2}(\tau)\right] R^{-1}(\tau) d \tau
$$

Solving Eq. (20) for $C$ gives

$$
C=\frac{f_{1}}{\int_{t}^{t_{f}} h_{1}(\tau)\left[h_{1}(\tau)-\lambda h_{2}(\tau)\right] R^{-1}(\tau) d \tau}
$$

Substituting Eq. (21) into Eq. (19) results in

$$
a_{M_{\sigma}}(\tau)=\frac{f_{1}\left[h_{1}(\tau)-\lambda h_{2}(\tau)\right] R^{-1}(\tau)}{\int_{t}^{t_{f}} h_{1}(\tau)\left[h_{1}(\tau)-\lambda h_{2}(\tau)\right] R^{-1}(\tau) d \tau}
$$

which is an incomplete guidance command due to the unknown Lagrange multiplier $\lambda$.

From Eq. (18), it can be obtained that the minimum value of the performance index as

$$
J=\frac{\left(f_{1}-\lambda f_{2}\right)^{2}}{2 \int_{t}^{t_{f}}\left[h_{1}(\tau)-\lambda h_{2}(\tau)\right]^{2} R^{-1}(\tau) d \tau}=\frac{\left(f_{1}-\lambda f_{2}\right)^{2}}{2\left(g_{1}-2 \lambda g_{12}+\lambda^{2} g_{2}\right)}
$$

where

$$
\begin{aligned}
g_{1} & =\int_{t}^{t_{f}} h_{1}^{2}(\tau) R^{-1}(\tau) d \tau \\
g_{12} & =\int_{t}^{t_{f}} h_{1}(\tau) h_{2}(\tau) R^{-1}(\tau) d \tau \\
g_{2} & =\int_{t}^{t_{f}} h_{2}^{2}(\tau) R^{-1}(\tau) d \tau
\end{aligned}
$$

In order to find the optimal Lagrange multiplier $\lambda$, we impose the condition $d J / d \lambda=0$ on Eq. (23) and then one can easily obtain that

$$
\lambda=\frac{f_{1} g_{12}-f_{2} g_{1}}{f_{1} g_{2}-f_{2} g_{12}}
$$

Substituting Eq. (25) into Eq. (22) gives the optimal guidance command as

$$
a_{M_{\sigma}}(\tau)=\frac{\left\{f_{1} h_{1}(\tau) g_{2}-\left[f_{2} h_{1}(\tau)+f_{1} h_{2}(\tau)\right] g_{12}+f_{2} h_{2}(\tau) g_{1}\right\} R^{-1}(\tau)}{g_{1} g_{2}-g_{12}^{2}}
$$

Using Eqs. (9), (13), and (14), the optimal guidance command can be formulated in the ZEM format as

$$
a_{M_{\sigma}}=k_{1} \frac{\int z d t}{t_{g o}^{3}}+k_{2} \frac{z}{t_{g o}^{2}}
$$


where

$$
\begin{aligned}
& k_{1}=\left(\frac{g_{2} t_{g o}^{5}-g_{12} t_{g o}^{4}}{g_{1} g_{2}-g_{12}^{2}}\right) R^{-1}(\tau) \\
& k_{2}=\left(\frac{g_{2} t_{g o}^{5}-2 g_{12} t_{g o}^{4}+g_{1} t_{g o}^{3}}{g_{1} g_{2}-g_{12}^{2}}\right) R^{-1}(\tau)
\end{aligned}
$$

To shape the guidance command, we consider a time-to-go weighting function as $R^{-1}(t)=t_{g o}^{N}$ with $N \geq 0$ [1], [20], [21]. Accordingly, if the value of $N$ is chosen as $N=0$, the resulting optimal guidance law is for energy consumption minimization. Additionally, we can safely predict that the designed guidance law with $N>0$ guarantees a small terminal acceleration command since the weighting function with $N>0$ becomes infinite as $t_{g o}$ approaches 0 . This property provides the missile with guaranteed operational margins to cope with the undesired disturbances when the missile approaches the target. Obviously, one can utilize other weighting functions to further shape or distribute the guidance command during the terminal homing phase. Substituting $R^{-1}(t)=t_{g o}^{N}$ into Eq. (28) gives

$$
k_{1}=(N+4)(N+5), \quad k_{2}=2(N+4)
$$

In this case, the proposed guidance command can then be obtained as

$$
a_{M_{\sigma}}=(N+4)(N+5) \frac{\int z d t}{t_{g o}^{3}}+2(N+4) \frac{z}{t_{g o}^{2}}
$$

Obviously, the term $2(N+4) z / t_{\text {go }}^{2}$ is the well-known PNG with a navigation ratio $2(N+4)$, whereas the term $(N+4)(N+5) \int z d t / t_{g o}^{3}$ is the integration of the ZEM to reduce the sensitivity to the unknown disturbances, e.g., target maneuvers. This property is totally different from previous guidance laws, where the information on the target maneuver is required for the implementation, and will be further analyzed in the following section.

\section{B. Implementation Issue}

For the implementation, it would be better to express the proposed guidance law in terms of the measurements of on-board seekers. From Eqs. (5) and (6), we can determine the kinematics relationship between the LOS rate and the ZEM as

$$
z=\dot{\sigma} V_{c} t_{g o}^{2}
$$

Substituting Eq. (31) into Eq. (30) gives the explicit guidance command as

$$
a_{M_{\sigma}}=(N+4)(N+5) \frac{\int\left(\dot{\sigma} V_{c} t_{g o}^{2}\right) d t}{t_{g o}^{3}}+2(N+4) V_{c} \dot{\sigma}
$$

Note that this guidance command is given by the function of closing velocity, LOS rate, and time-to-go. Therefore, the proposed guidance law requires only one additional information, e.g., the time-to-go, compared to the conventional PNG. This means that the proposed guidance law is also easy for practical implementation. For the implementation, the closing velocity and the LOS rate can be obtained from on-board seekers with a dedicated homing filter. In practice, the time-to-go information can be determined by using the conventional approximation method as

$$
t_{g o} \approx r / V_{c}
$$


Note that although the time-to-go estimation, shown in Eq. (33), tends to be smaller than the true time-to-go during the initial flight period, especially for large heading angle scenarios [21]-[24], this time-to-go estimation error gradually converges to zero as the missile approaches the target. Also, in the proposed guidance law, the timeto-go information is only used for computing the integral term, which is utilized to reduce the sensitivity against the unknown target acceleration. This implies that the time-to-go estimation error may degrade the robustness against the unknown target acceleration initially, but the degradation of final intercept accuracy due to this time-to-go estimation error is negligible in practice.

\section{Analysis of the Proposed Optimal Guidance LaW}

In this section, we will analyze the properties of the proposed guidance law. First, we discuss the ZEM dynamics and the closed-form solution of the guidance command to provide better understanding of the proposed guidance law. Then, the working principle of the proposed guidance law is investigated by comparing it with the adaptive guidance law.

\section{A. Behavior of the ZEM Dynamics}

Substituting Eq. (30) into Eq. (7) gives the second-order ZEM dynamics as

$$
\dot{z}+k_{2} \frac{z}{t_{g o}}+k_{1} \frac{\int z d t}{t_{g o}^{2}}=t_{g o} a_{T \sigma}
$$

The left-hand side of Eq. (34) represents the characteristic equation of the ZEM dynamics under the proposed guidance law. Additionally, the right-hand side of Eq. (34) is the external forcing term to the ZEM dynamics. From Eq. (34), we can determine the natural frequency $\omega$ and damping ratio $\zeta$ of the ZEM dynamics as

$$
\omega=\frac{\sqrt{k_{1}}}{t_{g o}}, \quad \zeta=\frac{k_{2}}{2 \sqrt{k_{1}}}
$$

which reveals that

$$
\frac{\sqrt{k_{1}}}{t_{f}} \leq \omega<\infty
$$

The preceding inequality shows that the bandwidth of the guidance loop increases to infinite under the proposed guidance law when the interceptor approaches the target. Also, we can readily observe that the damping ratio is proportional to the value of $k_{2}$, which is the guidance gain of the PNG term.

\section{B. Closed-Form Solution of the Proposed Guidance Law}

To further analyze the characteristics of the ZEM, we seek to find its closed-form solution. It can be noted that Eq. (34) is a second-order Cauchy-Euler differential equation with respect to $t_{g o}$. By considering $\int z d t=c t_{g o}^{n}$ as a fundamental solution of Eq. (34), where $c$ and $n$ are two nonzero constants, one can readily derive the homogeneous solution as

$$
\int z d t=c_{1} t_{g o}^{N+5}+c_{2} t_{g o}^{N+4}
$$

where $c_{1}, c_{2}$ are two constants governed by the initial conditions. 
Additionally, by assuming $a_{T_{\sigma}}$ is constant and considering $\int z d t=p t_{g o}^{3}$ as a particular solution of Eq. (34), where $p$ is a nonzero constant, one can obtain the particular solution as

$$
\int z d t=\frac{a_{T_{\sigma}}}{(N+1)(N+2)} t_{g o}^{3}
$$

Then, combining Eqs. (37) and (38) gives the closed-form solution of the integral ZEM as

$$
\int z d t=c_{1} t_{g o}^{N+5}+c_{2} t_{g o}^{N+4}+\frac{a_{T_{\sigma}}}{(N+1)(N+2)} t_{g o}^{3}
$$

Applying the initial boundary conditions (i.e., $z(0)=z_{0}$ and $\int z d t=0$ ) on the generic solution Eq. (39) generates

$$
\begin{gathered}
c_{1}=-z_{0} t_{f}^{-N-4}+\frac{a_{T_{\sigma}}}{N+2} t_{f}^{-N-2} \\
c_{2}=z_{0} t_{f}^{-N-3}-\frac{a_{T_{\sigma}}}{N+1} t_{f}^{-N-1}
\end{gathered}
$$

Differentiating Eq. (39) with respect to time gives the closed-form ZEM as

$$
z=-c_{1}(N+5) t_{g o}^{N+4}-c_{2}(N+4) t_{g o}^{N+3}-\frac{3 a_{T_{\sigma}}}{(N+1)(N+2)} t_{g o}^{2}
$$

which clearly reveals that the ZEM can converge to zero as the missile approaches the target, $t_{g o} \rightarrow 0$, even in the presence of unknown target accelerations. Therefore, unlike to previous PI guidance laws [18], [19], the proposed guidance law can rigorously guarantee the finite time convergence. Also, from Eq. (42), we can readily observe that the effect of unknown target acceleration on the ZEM can be mitigated as $N$ increases.

Substituting Eq. (39) and Eq. (42) into Eq. (30) results in

$$
a_{M_{\sigma}}=-c_{1}(N+4)(N+5) t_{g o}^{N+2}-c_{2}(N+3)(N+4) t_{g o}^{N+1}+\frac{(N+4)(N-1)}{(N+1)(N+2)} a_{T_{\sigma}}
$$

which shows that the guidance command converges to a specific value at the time of impact once as

$$
a_{M_{\sigma}}\left(t_{f}\right)=\frac{(N+4)(N-1)}{(N+1)(N+2)} a_{T_{\sigma}}
$$

As shown in Eq. (44), the magnitude of the final guidance command is governed by $N$ and the magnitude of target acceleration. Therefore, we can readily predict that the proposed guidance command converges to zero under $N \geq 0$ for a non-maneuvering target (i.e., $a_{T_{\sigma}}=0$ ). Additionally, taking the derivative of $a_{M_{\sigma}}$ with respect to time, one can imply that

$$
\dot{a}_{M_{\sigma}}=c_{1}(N+2)(N+4)(N+5) t_{g o}^{N+1}+c_{2}(N+1)(N+3)(N+4) t_{g o}^{N}
$$

Solving $\dot{a}_{M_{\sigma}}=0$ for $t_{g o}$ gives

$$
t_{g o, m}=-\frac{c_{2}(N+1)(N+3)}{c_{1}(N+2)(N+5)}
$$

which reveals that the guidance command takes its maximum magnitude either at the boundary points or $t_{g o}=t_{g o, m}$. This information is helpful in finding the required acceleration demand with physical constraints. 


\section{Sensitivity to Unknown Target Acceleration}

This subsection reveals how the proposed guidance law can be used to reduce the sensitivity against unknown target maneuvers (i.e., the working principle of the proposed guidance law). Especially, the physical meaning of the integral term in the guidance command is analyzed by introducing an alternative form of the proposed guidance law. To this end, substituting Eq. (30) into Eq. (7) gives the closed-loop ZEM dynamics as

$$
\dot{z}=-t_{\text {go }}\left(k_{2} \frac{z}{t_{\text {go }}^{2}}+k_{1} \frac{\int z d t}{t_{\text {go }}^{3}}\right)+t_{g o} a_{T_{\sigma}}
$$

For notation simplicity, let us define the equivalent proportional gain $k_{P}$, the equivalent integral gain $k_{I}$, and the control input gain $b$, as

$$
k_{P}=\frac{k_{2}}{t_{g o}^{2}}, \quad k_{I}=\frac{k_{1}}{t_{g o}^{3}}, \quad b=t_{g o}
$$

Then, by using these variables, the closed-loop ZEM dynamics shown in Eq. (47) can be rewritten as

$$
\dot{z}=-b\left(k_{P} z+k_{I} \int z d t\right)+b a_{T_{\sigma}}
$$

Since the gains $k_{P}, k_{I}$ and $b$ are time-varying due to the time-to-go, we utilize the widely-accepted instantaneous linear time-invariant system concept for analysis. In other words, we assume that $k_{P}, k_{I}$ and $b$ are piece-wise constants. Note that this assumption is widely-used in guidance loop analysis. Next, let us define a new variable as

$$
\hat{a}_{T_{\sigma}}=k_{I} \int z d t
$$

Then, the ZEM dynamics (49) can be rearranged using this variable as

$$
\dot{z}=-b k_{P} z-b \hat{a}_{T_{\sigma}}+b a_{T_{\sigma}}
$$

Additionally, by the instantaneous linear time-invariant system concept, the time-derivation of Eq. (50) can be determined as

$$
\dot{\hat{a}}_{T_{\sigma}}=k_{I} z
$$

In the guidance problem, since zero ZEM will lead to a perfect interception, the desired value of the ZEM can be written as $z_{d}=0$. Then, by using a slack variable $z_{d}$, Eq. (52) can be rewritten as

$$
\dot{\hat{a}}_{T_{\sigma}}=k_{I}\left(z-z_{d}\right)
$$

From Eq. (53), the term $\left(z-z_{d}\right)$ can be considered as the ZEM tracking error between the current value and the desired value. Therefore, from Eqs. (51) and (53), one can clearly observe that the proposed optimal PI guidance law can be interpreted as an instantaneous direct model reference adaptive guidance law. In the proposed guidance law, $z_{d}=0$ is considered as the reference model and $k_{I}$ can be regarded as the adaptive gain. Compared to the adaptive guidance law, the term, corresponding to the adaptive gain, is given by a time-varying function of the time-to-go in the proposed guidance law. Based on this observation, we can readily predict that the integral term in the optimal PI guidance law plays the same role as the adaptive/update law in adaptive guidance law. This is why the PI guidance law can be utilized to attenuate the effect of the unknown target maneuver: the integral term, acting as an instantaneous direct adaptive law, estimates the unknown target maneuver online and the estimated signal is 
feedback to the guidance command to attenuate the effect of target maneuvers. In conclusion, the proposed optimal PI guidance can be regarded as a special case of adaptive guidance with a specified reference model.

Hereafter, we discuss the boundedness of the closed-loop guidance system. We define $\tilde{a}_{T \sigma}=a_{T \sigma}-\hat{a}_{T_{\sigma}}$ as the estimation error of the adaptive signal and consider the following Lyapunov function candidate

$$
\Omega=z^{2}+\frac{b}{k_{I}} \tilde{a}_{T \sigma}^{2}
$$

Assume that the target maneuver is slowly-varying with respect to time, i.e., $\dot{a}_{T \sigma}=0$, differentiating $\Omega$ with respect to time yields

$$
\begin{aligned}
\dot{\Omega} & =2 z \dot{z}+\frac{2 b}{k_{I}} \tilde{a}_{T_{\sigma}} \dot{\tilde{a}}_{T_{\sigma}} \\
& =2 z\left(-b k_{P} z+b \tilde{a}_{T_{\sigma}}\right)-\frac{2 b}{k_{I}} \tilde{a}_{T_{\sigma}} \dot{\hat{a}}_{T_{\sigma}} \\
& =2 z\left(-b k_{P} z+b \tilde{a}_{T_{\sigma}}\right)-2 b \tilde{a}_{T_{\sigma}} z \\
& =-2 b k_{P} z^{2} \\
& \leq 0
\end{aligned}
$$

where the fact $b k_{P}>0$ is used in the derivation. The preceding inequality proves that the ZEM and the target maneuver estimation errors are uniformly ultimately bounded. Application of Barbalat's lemma [25] reveals that the ZEM can converge to zero even in the presence of target maneuvers. From the above Lyapunov analysis, we notice that the ZEM can be upper bounded by

$$
\|z\| \leq \sqrt{\Omega} \leq \sqrt{\Omega(0)}=\sqrt{z^{2}(0)+\frac{b}{k_{I}} \tilde{a}_{T \sigma}^{2}(0)}
$$

This bound reveals that the effect of target maneuver on ZEM can be reduced by increasing the integral gain $k_{I}$. This coincides with the property of adaptive gain in adaptive control. Furthermore, since the integral gain $k_{I}$ increases as time goes in the proposed guidance law, fast adaptation is guaranteed as the missile approaches the target. In the analysis, the results of sensitivity to target maneuvers under the proposed optimal PI guidance law are derived based on the instantaneous linear time-invariant system concept for the purpose of analysis. Therefore, the presented results may not be exactly the same as the practical ones, but the tendency of the presented results is still valid.

The findings in this subsection have potential importance. First, the results give us better understanding of how PI guidance law works in maneuvering target interception through the integral action. In practice, such information is important in ensuring confidence in the performance and reliability of the guidance law when implementing it in a real system. Second, the results can also provide a point of connection between the PI guidance and adaptive guidance. This allows us to have better understanding and utilization of both guidance laws. For example, we can design new PI guidance laws that provide better performance through appropriate choice of the reference model, like the approaches in modified adaptive control.

\section{Simulation Results}

In this section, the effectiveness of the proposed optimal PI guidance law is validated through nonlinear numerical simulations. We first investigate the fundamental characteristics of the proposed guidance law. Then, the capability 
TABLE I: Initial conditions for homing engagement.

\begin{tabular}{cc}
\hline \hline Parameters & Values \\
\hline Missile initial position, $\left(x_{M}(0), y_{M}(0)\right)$ & $(200 \mathrm{~m}, 3200 \mathrm{~m})$ \\
Missile flight velocity, $V_{M}$ & $1000 \mathrm{~m} / \mathrm{s}$ \\
Missile initial flight path angle, $\gamma_{M}(0)$ & $0^{\circ}$ \\
Target initial position, $\left(x_{T}(0), y_{T}(0)\right)$ & $(1000 \mathrm{~m}, 3100 \mathrm{~m})$ \\
Target velocity, $V_{T}$ & $600 \mathrm{~m} / \mathrm{s}$ \\
Target initial flight path angle, $\gamma_{T}(0)$ & $30^{\circ}$ \\
\hline \hline
\end{tabular}

TABLE II: Control effort for various guidance gains.

\begin{tabular}{ccccc}
\hline \hline & $N=0$ & $N=1$ & $N=2$ & $N=3$ \\
\hline Control effort & 23044 & 24862 & 27337 & 29576 \\
\hline \hline
\end{tabular}

of reducing the sensitivity to the unknown target maneuver is tested and compared with the benchmark PNG law and APNG. Finally, the proposed optimal PI guidance is compared with other PI guidance laws. In all simulations, we consider a fast moving target interception scenario. The initial conditions for the considered scenario are summarized in Table I.

\section{A. Characteristics of the Proposed Guidance Law}

This simulation in this subsection investigates the basic characteristics of the proposed guidance law with various guidance gains $N=0,1,2,3$. For this purpose, we assume that the target performs no evasive maneuvers in this case. Fig. 2 compares the patterns of state variables with various guidance gains. From this figure, it can be noted that the ZEM can converge to zero at the time of impact and therefore successful interception is guaranteed. This result matches with the analytical results of the closed-form solution. The results in Fig. 2 also clearly reveal that the proposed guidance law with larger guidance gain $N$ leads to faster convergence speed of the ZEM. The response of the integral ZEM $\int z d t$ with various guidance gains is presented in Fig. 3, which shows that the terminal constraint $\int_{0}^{t_{f}} z d t=0$ is satisfied by the proposed guidance law. Furthermore, Fig. 3 also reveals that the variation of the ZEM can be reduced by increasing the guidance gain $N$, leading to the improved robustness against external disturbances. Figure 4 provides the required guidance commands for various guidance gains. Due to physical limits, we enforce hard constraint $\left|a_{M}\right| \leq 400 \mathrm{~m} / \mathrm{s}^{2}$ in the simulations. The corresponding control efforts, defined as $\int_{0}^{t_{f}} a_{M}^{2}(\tau) d \tau$, for various guidance gains are summarized in Table II. One can note from Fig. 3 that the proposed guidance law shown with $N \geq 0$ guarantees zero guidance command at the time of impact for a non-maneuvering target as expected in the closed-form solution. However, increasing the guidance gain $N$ requires higher acceleration command during the initial flight period, thereby generating more control effort, as shown in Table II. 


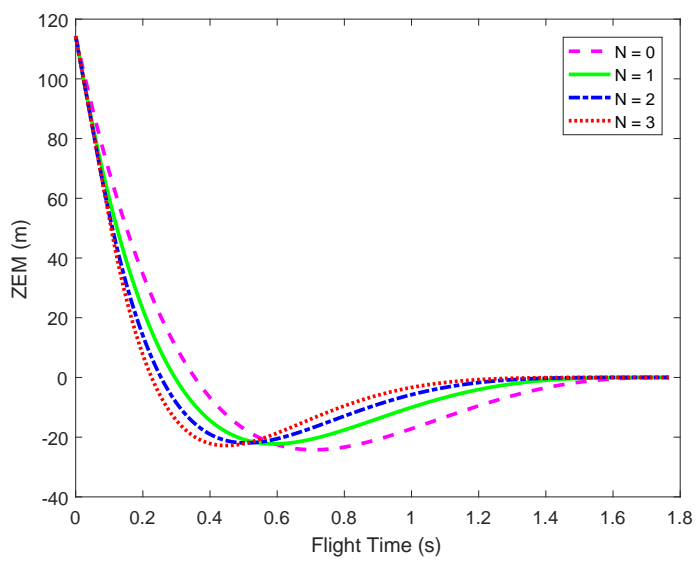

Fig. 2: ZEM with various guidance gains.

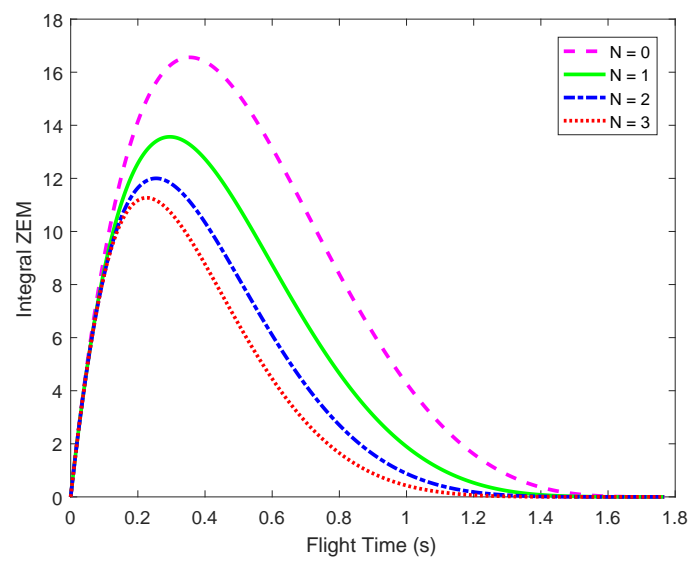

Fig. 3: Integral ZEM with various guidance gains.

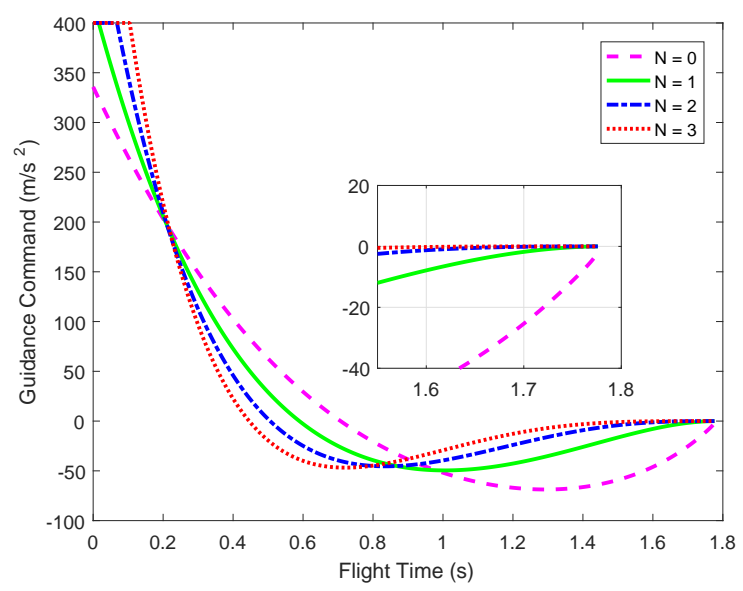

Fig. 4: Acceleration command with various guidance gains.

\section{B. Reduced Sensitivity to Unknown Target Maneuvers}

The simulation in this subsection investigates the robustness against unknown target maneuvers under the proposed optimal PI guidance law. To justify the robustness of the proposed guidance scheme, two different cases of target maneuvers are considered: (1) $a_{T}=300 \mathrm{~m} / \mathrm{s}^{2}$; and (2) $a_{T}=-300 \mathrm{~m} / \mathrm{s}^{2}$. For better illustration, we compare the proposed guidance law with the benchmark PNG and APNG. The acceleration command of the PNG law is given by

$$
a_{M_{\sigma}}=(N+3) V_{c} \dot{\sigma}
$$

which is proved to be an optimal guidance law that minimizes the performance index

$$
J=\int_{t}^{t_{f}} \frac{a_{M_{\sigma}}^{2}(\tau)}{\left(t_{f}-\tau\right)^{N}} d \tau
$$

The acceleration command of the APNG law is defined as [1]

$$
a_{M_{\sigma}}=(N+3) V_{c} \dot{\sigma}+\frac{(N+3)}{2} a_{T_{\sigma}}
$$


TABLE III: Miss distance and control effort of different guidance laws.

\begin{tabular}{cccccc}
\hline \hline & Scenario & PNG5 & PNG10 & Proposed PI & APNG \\
\hline \multirow{2}{*}{ Miss distance } & Case1 & $2.018 m$ & $1.114 m$ & $0.743 m$ & $0.651 m$ \\
& Case2 & $30.669 m$ & $1.554 m$ & $0.914 m$ & $0.663 m$ \\
\hline \multirow{2}{*}{ Control effort } & Case 1 & 125582 & 92221 & 84605 & 83511 \\
& Case 2 & 200028 & 190457 & 151577 & 50864 \\
\hline \hline
\end{tabular}

In the simulations, two different PNG laws are utilized: (1) PNG5 with $N=2$; and (2) PNG10 with $N=7$. The guidance gain of the proposed guidance law and APNG is chosen as $N=2$. Note that the proposed guidance law with $N=2$ minimizes the same performance index as PNG5 and APNG with $N=2$. From (59), note that the implementation of the APNG law requires the information on target maneuver, which is an ideal case.

The interception trajectories for cases 1 and 2, obtained from these four different guidance laws, are presented in Fig. 5 (a) and (b). The recorded miss distance and control effort are summarized in Table III. Fig. 5 (a) and (b) show that both PNG10 and the proposed optimal PI guidance law successfully intercept the target in both cases with guaranteed miss distance, whereas the missile guided by PNG5 misses the target in case 2. Moreover, the recorded miss distance, provided in Table III, reveals that the proposed guidance law exhibits better performance in terms of the interception accuracy. The responses of ZEM for cases 1 and 2 under different guidance laws are presented in Fig. 5 (c) and (d). From these two figures, it can be noted that the ZEM under the proposed optimal PI guidance law as well as the PNG10 law can converge to zero at the time of impact to guarantee successful interception and has a divergent pattern under the PNG5 law for case 2. Fig. 5 (e) and (f) provide the acceleration command of different guidance laws for both cases. Compared to the PNG10 law, the proposed guidance law requires relatively higher acceleration command at the initial flight phase but the demand command converges to a low level when the interceptor approaches the target. Furthermore, the recorded control efforts, given in Table III, reveal that the proposed optimal PI guidance law requires less energy consumption than the other two laws in the two considered scenarios. From the results, shown in Table III, one can note that APNG achieves the best performance in terms of miss distance and control effort. However, APNG is an ideal law that is limited to implement in real applications due to the requirement of accurate target maneuver information. Compared to two PNG laws, the performance of the proposed optimal PI guidance law is comparable to that of the APNG and it is not required target maneuver information, which demonstrates the superiority of the proposed approach.

\section{Comparison with Previous PI Guidance Laws}

To further show the superiority and gain insight of the proposed guidance law, comparisons with previous PI guidance laws [18], [19] are performed in a same scenario in this subsection. In [18], the PI guidance was intuitively formulated using PI-control concept as

$$
a_{M_{\sigma}}=N_{p} V_{c} \dot{\sigma}+N_{I} V_{c} \sigma
$$

where $N_{p}$ and $N_{I}$ are the user-defined constant guidance gains. 


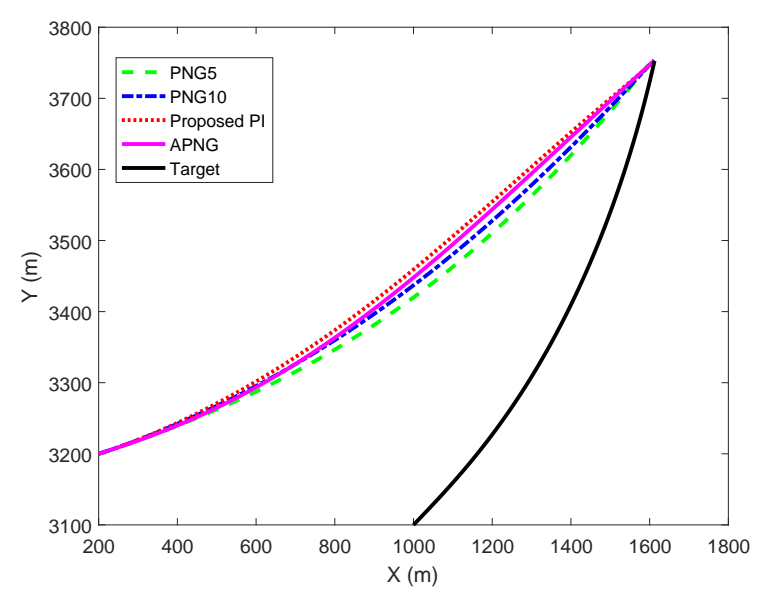

(a) Interception trajectory of case 1

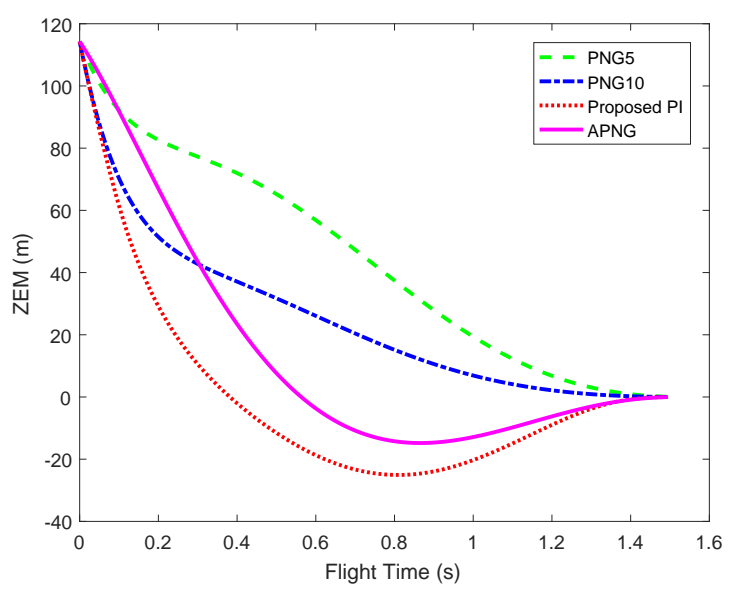

(c) ZEM of case 1

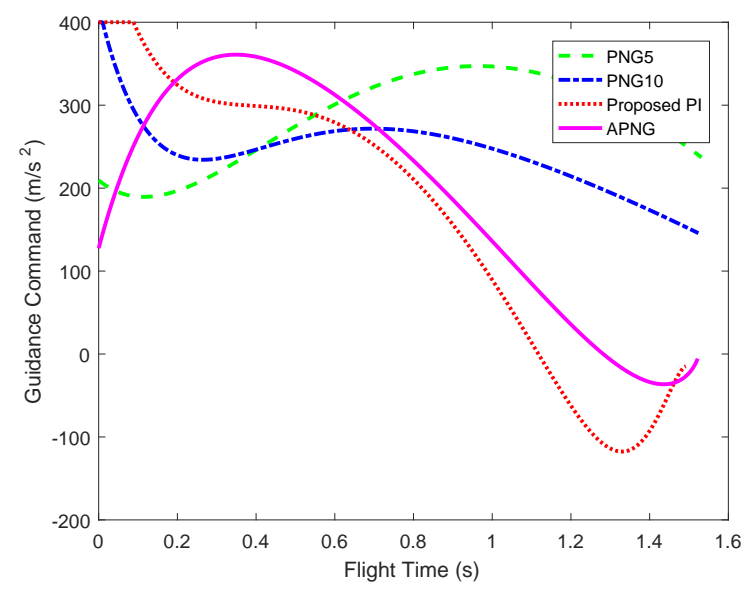

(e) Acceleration command of case 1

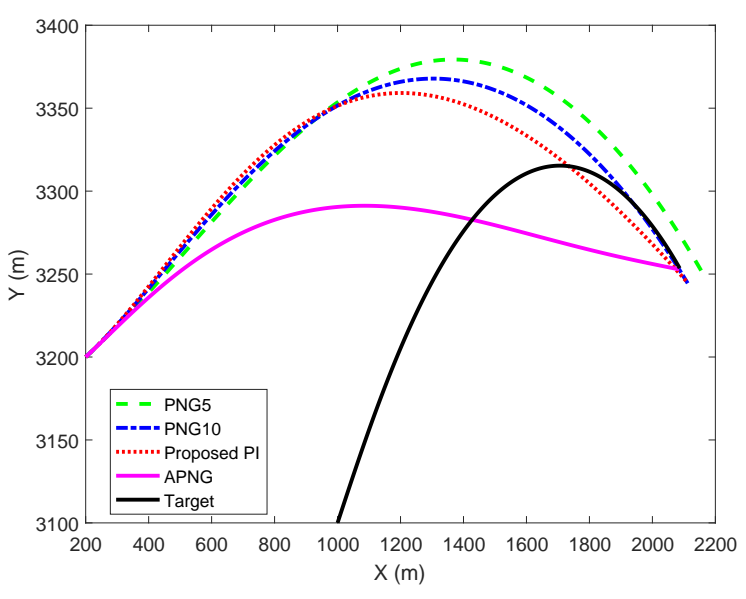

(b) Interception trajectory of case 2

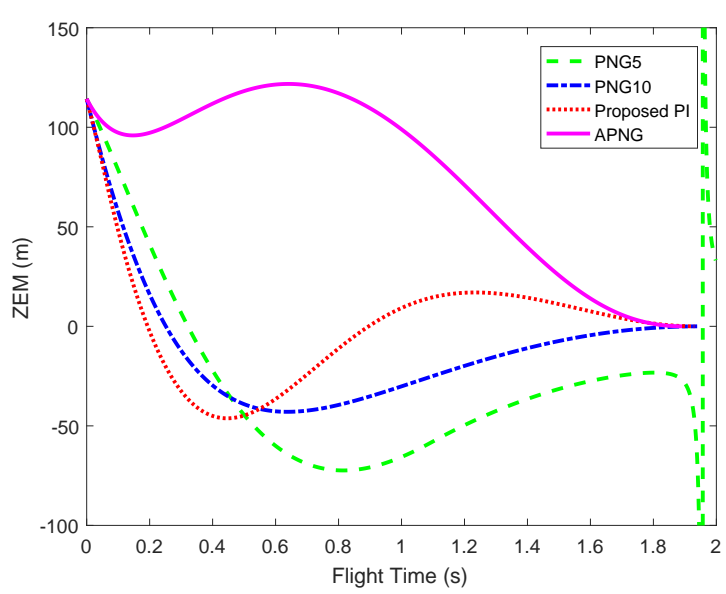

(d) ZEM of case 2

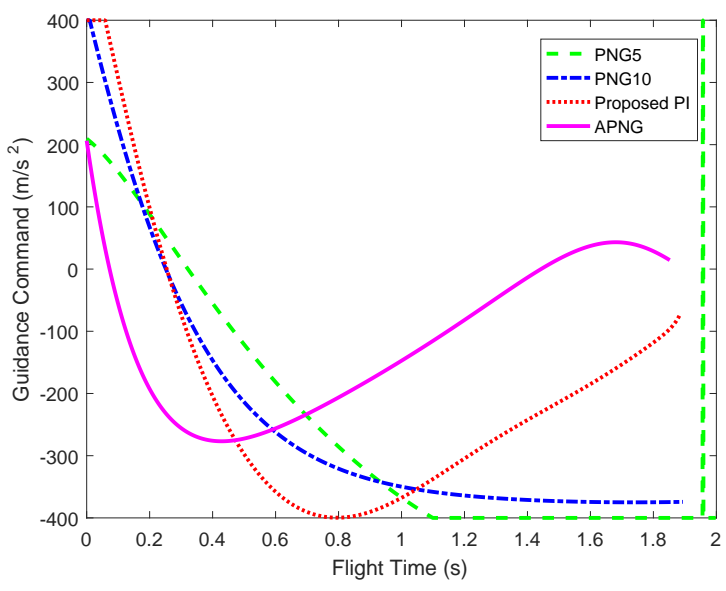

(f) Acceleration command of case 2

Fig. 5: Comparison results of different guidance laws. 
Also, the authors in [19] further developed an optimal PI guidance law based on the kinematics ignoring the target maneuver term as

$$
\begin{aligned}
& \dot{x}_{1}=x_{2} \\
& \dot{x}_{2}=-\frac{2 \dot{r}}{r} x_{2}-\frac{1}{r} a_{M_{\sigma}}
\end{aligned}
$$

where $x_{1}=\sigma$ and $x_{2}=\dot{\sigma}$. The guidance command proposed in [19] is given by

$$
a_{M_{\sigma}}=K_{P}(t) \dot{\sigma}+K_{I}(t) \sigma
$$

where the time-varying gains $K_{P}(t)$ and $K_{I}(t)$ are obtained by solving a infinite time linear quadratic regulation (LQR) problem at each step with the cost function

$$
J=\int_{0}^{\infty}\left(x^{T} Q x+a_{M_{\sigma}}^{T} R a_{M_{\sigma}}\right) d t
$$

where $Q$ and $R$ are the user-defined positive definite matrices.

It follows from Eq. (60) and Eq. (62) that PI guidance law, shown in Eq. (60), is a special form of the optimal PI guidance law shown in Eq. (62). Therefore, we only compare the proposed PI guidance law with the optimal PI guidance law Eq. (62). It is known that, in LQR control, increasing the value of the diagonal elements of $Q$ increases the penalty on the system states while increasing $R$ helps to reduce the control effort. Therefore, we utilize different $Q$ and $R$ for the implementation of guidance law Eq. (62) to demonstrate the effectiveness of the proposed approach: (1) PI guidance 1: $Q=10^{4} I, R=1$; (2) PI guidance 2: $Q=10^{6} I, R=1$; and (3) PI guidance 3: $Q=10^{6} I, R=10$.

The simulations results, including interception trajectory, ZEM profile and guidance command with $a_{T}=$ $-300 \mathrm{~m} / \mathrm{s}^{2}$ are presented in Figs. 6-8. The recorded miss distance and control effort are summarized in Table IV. From these results, it is clear that both PI guidance law 2 and the proposed guidance law can intercept the target successfully while other two PI guidance laws miss the target due to limited maneuverability of the interceptor. Additionally, the proposed guidance law can reduce the required acceleration command when the missile approaches the target and the control effort during the entire engagement. Since kinematics Eq. (61) is nonlinear and timevarying, it is intractable to derive a closed-form solution for $K_{P}(t)$ and $K_{I}(t)$. Therefore, implementing optimal PI guidance law Eq. (62) requires solving a LQR problem at every time instant, which is time-consuming for the onboard flight computer. As a comparison, the proposed guidance law is given in an explicit form of the measured information and thus is more suitable for practical application. Another issue of the optimal PI guidance law Eq. (62), as stated in [19], is that there is no theoretical way to choose proper $Q$ and $R$. In contrary, the guidance gain $N$ of the proposed PI guidance law can be easily selected to achieve desired convergence rate of the ZEM by utilizing the closed-form solution, as we discussed in the previous section.

\section{CONCLusions}

This paper proposes a novel optimal guidance law using PI control concept to reduce the sensitivity to the unknown target maneuvers. We first augment the integral ZEM as a new system state and formulate a finite time regulation problem for the augmented system. Based on this result, a time-to-go weighted optimal guidance law is 
TABLE IV: Miss distance and control effort of different guidance laws.

\begin{tabular}{lcccc}
\hline \hline & PI Guidance 1 & PI Guidance 2 & PI Guidance 3 & Proposed PI \\
\hline Miss distance & $27.127 m$ & $0.981 m$ & $17.003 m$ & $0.914 m$ \\
Control effort & 191581 & 167722 & 180454 & 151577 \\
\hline \hline
\end{tabular}

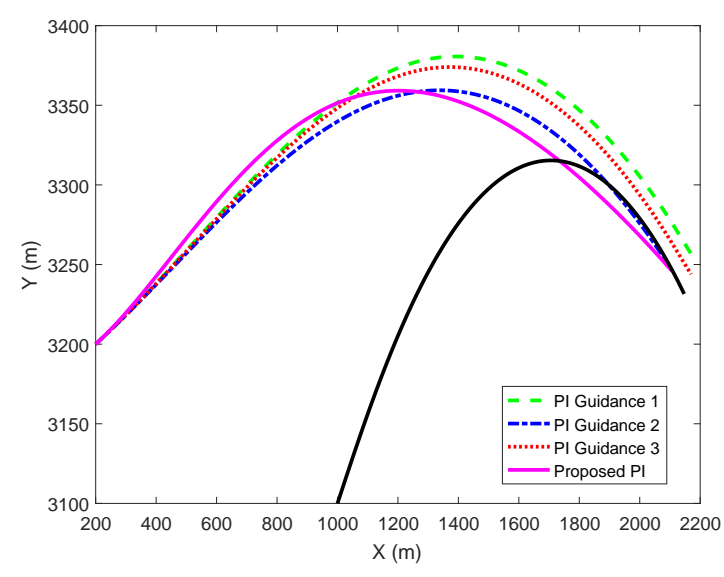

Fig. 6: The interception trajectory of different guidance

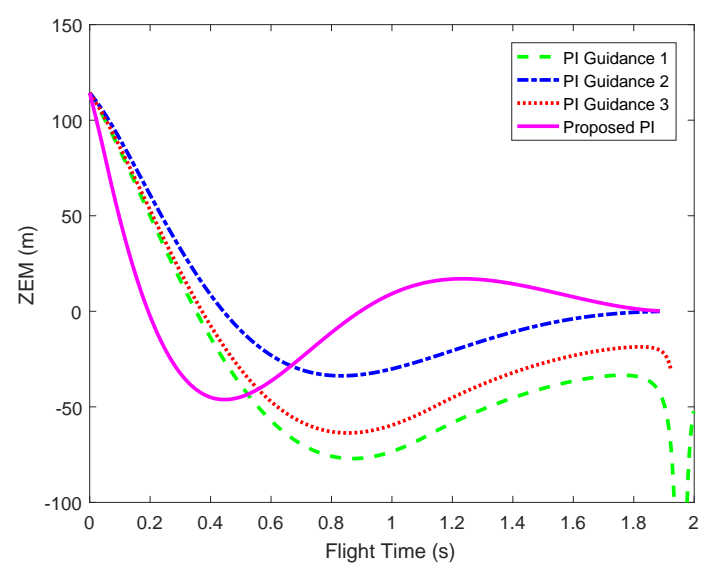

Fig. 7: The ZEM with various guidance laws. laws.

derived through the Schwarz's inequality approach. The final guidance command is shown to be a PI form with time-varying gains in the ZEM format, where the proportional term is the well-known PNG to nullify the ZEM and the integral term is an additional guidance command to reduce the sensitivity against the disturbance (i.e., unknown target maneuver). The closed-form solution and the closed-loop ZEM dynamics under the proposed guidance law are determined to investigate the properties of the proposed guidance law. Additionally, by introducing an alternative form of the guidance command, the working principle of the proposed optimal PI guidance law is analyzed and the results reveal that the proposed guidance law is exactly the same as an instantaneous model reference adaptive guidance law with a specified reference model. Future work includes extending the proposed guidance approach to impact angle control.

\section{REFERENCES}

[1] P. Zarchan, Tactical and strategic missile guidance. American Institute of Aeronautics and Astronautics, 2012.

[2] S. A. Murtaugh and H. E. Criel, "Fundamentals of proportional navigation,” IEEE spectrum, vol. 3, no. 12, pp. 75-85, 1966.

[3] M. Guelman, "Proportional navigation with a maneuvering target," IEEE Transactions on Aerospace and Electronic Systems, no. 3, pp. 364-371, 1972.

[4] P.-J. Yuan and S.-C. Hsu, "Solutions of generalized proportional navigation with maneuvering and nonmaneuvering targets," IEEE Transactions on Aerospace and Electronic Systems, vol. 31, no. 1, pp. 469-474, 1995.

[5] P. Mahapatra and U. Shukla, "Accurate solution of proportional navigation for maneuvering targets," IEEE Transactions on Aerospace and Electronic Systems, vol. 25, no. 1, pp. 81-89, 1989.

[6] F. W. Nesline and P. Zarchan, "A new look at classical vs modern homing missile guidance," Journal of Guidance, Control, and Dynamics, vol. 4, no. 1, pp. 78-85, 1981. 


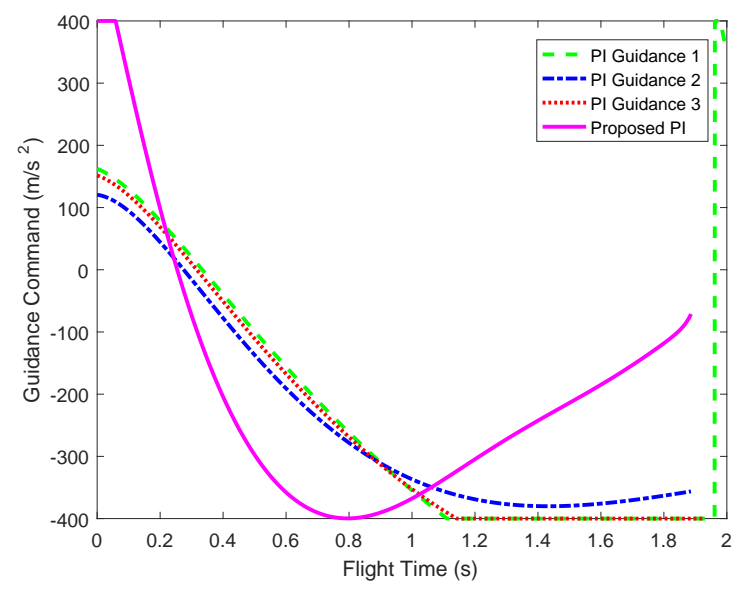

Fig. 8: The acceleration command of different guidance laws.

[7] I.-J. Ha, J.-S. Hur, M.-S. Ko, and T.-L. Song, "Performance analysis of png laws for randomly maneuvering targets," IEEE Transactions on Aerospace and Electronic Systems, vol. 26, no. 5, pp. 713-721, 1990.

[8] G. Weiss and I. Rusnak, "All-aspect three-dimensional guidance law based on feedback linearization," Journal of Guidance, Control, and Dynamics, vol. 38, no. 12, pp. 2421-2428, 2015.

[9] N. Cho and Y. Kim, "Optimality of augmented ideal proportional navigation for maneuvering target interception," IEEE Transactions on Aerospace and Electronic Systems, vol. 52, no. 2, pp. 948-954, 2016.

[10] K. R. Babu, I. Sarma, and K. Swamy, "Switched bias proportional navigation for homing guidance against highly maneuvering targets," Journal of Guidance, Control, and Dynamics, vol. 17, no. 6, pp. 1357-1363, 1994.

[11] D. Chwa and J. Y. Choi, "Adaptive nonlinear guidance law considering control loop dynamics," IEEE Transactions on Aerospace and Electronic Systems, vol. 39, no. 4, pp. 1134-1143, 2003.

[12] D. Chwa, J. Y. Choi, and S. G. Anavatti, "Observer-based adaptive guidance law considering target uncertainties and control loop dynamics," IEEE Transactions on Control Systems Technology, vol. 14, no. 1, pp. 112-123, 2006.

[13] D. Zhou and B. Xu, "Adaptive dynamic surface guidance law with input saturation constraint and autopilot dynamics," Journal of Guidance, Control, and Dynamics, vol. 39, no. 5, pp. 1155-1162, 2016.

[14] D. Cho, H. J. Kim, and M.-J. Tahk, "Fast adaptive guidance against highly maneuvering targets," IEEE Transactions on Aerospace and Electronic Systems, vol. 52, no. 2, pp. 671-680, 2016.

[15] D. Chwa, "Robust nonlinear disturbance observer-based adaptive guidance law against uncertainties in missile dynamics and target maneuver," IEEE Transactions on Aerospace and Electronic Systems, 2018.

[16] D. Cho, H. J. Kim, and M.-j. Tahk, "Impact angle constrained sliding mode guidance against maneuvering target with unknown acceleration," IEEE Transactions on Aerospace and Electronic Systems, vol. 51, no. 2, pp. 1310-1323, 2015.

[17] S. He, T. Song, and D. Lin, "Impact angle constrained integrated guidance and control for maneuvering target interception," Journal of Guidance, Control, and Dynamics, vol. 40, no. 10, pp. 2653-2661, 2017.

[18] M. Golestani and I. Mohammadzaman, "Pid guidance law design using short time stability approach," Aerospace Science and Technology, vol. 43, pp. 71-76, 2015.

[19] Ç. Evcimen and K. Leblebicioğlu, "An adaptive, optimal proportional-integral guidance for missiles," in AIAA Guidance, Navigation, and Control Conference, 2013.

[20] C.-H. Lee, M.-J. Tahk, and J.-I. Lee, "Generalized formulation of weighted optimal guidance laws with impact angle constraint," IEEE Transactions on Aerospace and Electronic Systems, vol. 49, no. 2, pp. 1317-1322, 2013.

[21] C.-K. Ryoo, H. Cho, and M.-J. Tahk, “Time-to-go weighted optimal guidance with impact angle constraints," IEEE Transactions on Control Systems Technology, vol. 14, no. 3, pp. 483-492, 2006.

[22] A. Ratnoo and D. Ghose, "State-dependent riccati-equation-based guidance law for impact-angle-constrained trajectories," Journal of Guidance, Control, and Dynamics, vol. 32, no. 1, pp. 320-326, 2009. 
[23] Y.-I. Lee, S.-H. Kim, J.-I. Lee, and M.-J. Tahk, "Analytic solutions of generalized impact-angle-control guidance law for first-order lag system," Journal of Guidance, Control, and Dynamics, vol. 36, no. 1, pp. 96-112, 2013.

[24] C.-K. Ryoo, H. Cho, and M.-J. Tahk, "Optimal guidance laws with terminal impact angle constraint," Journal of Guidance Control and Dynamics, vol. 28, no. 4, pp. 724-732, 2005.

[25] H. K. Khalil, Noninear Systems. Prentice-Hall, New Jersey, 1996.

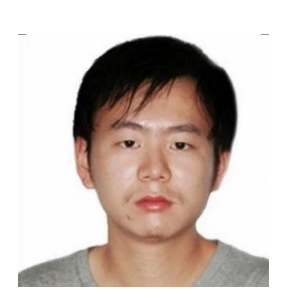

Shaoming He was born in 1991. He received his MSc degree in Aerospace Engineering from Beijing Institute of Technology in 2016. He is currently a PhD student in the School of Aerospace, Transport and Manufacturing, Cranfield University. His research interests include multi-target tracking, guidance and control.

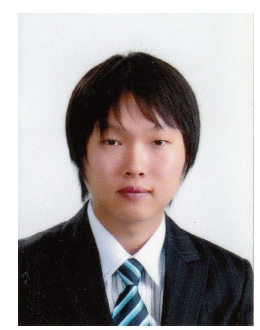

Chang-Hun Lee received the B.S., M.S., and Ph.D. degrees in aerospace engineering from Korea Advanced Institute of Science and Technology (KAIST), in 2008, 2010, and 2013, respectively.

From 2013 to 2015 he was a senior researcher for guidance and control (G\&C) team at the Agency for Defense Development (ADD) in Korea. Since 2016, he has worked for school of aerospace, transportation, and manufacturing at Cranfield University, UK where he is currently a research fellow. His recent research areas are advanced missile guidance and control, cooperative control for UAVs, target tracking filter, deep learning, and aviation data analytics. 
2018-04-09

\section{Optimal proportional-integral guidance with reduced sensitivity to target maneuvers}

He, Shaoming

IEEE

He S, Lee $\mathrm{C}-\mathrm{H}$, Optimal proportional-integral guidance with reduced sensitivity to target maneuvers, IEEE Transactions on Aerospace and Electronic Systems, Volume 54, Issue 5, October 2018, pp. 2568-2579

http://dx.doi.org/10.1109/TAES.2018.2824678

Downloaded from Cranfield Library Services E-Repository 